\title{
Umbilical Port Site Hernia and Diastasis Recti
}

\author{
Hyun Jeong Ki, M.D., Jun Beom Park, M.D., Ph.D., Ji-Young Sul, M.D., Ph.D. \\ Department of Surgery, Chungnam National University College of Medicine, Chungnam National University Hospital, Daejeon, Korea
}

\begin{abstract}
Purpose: The port site hernia (PSH) is a specific type of incisional hernia related to the trocar sites of laparoscopic surgery. Diastasis recti of the abdominis muscle (DR) is the separation of the rectus muscle by a certain distance. The present study aims to present our experience with umbilical PSH and concomitant DR and to raise awareness of DR as one of the risk factors of umbilical PSH.

Methods: Eighteen patients with umbilical PSH after laparoscopic abdominal surgery, was retrospectively reviewed. Preoperative CT was analyzed to measure the Inter-recti distance (IRD) for all patients. Other factors, such as trocar size, wound infection, obesity (BMI), port extension, suture materials, and pre-existing co-morbidities, were recorded and analyzed.

Results: Extension of the port incision was associated with umbilical PSH. Ten out of eighteen umbilical PSH patients (56\%) had DR before they had first laparoscopic surgery. Nine (50\%) patients showed sarcopenia. Moreover, four out of five recurrences had DR. More than two recurrences were all associated with DR.

Conclusion: Port extension and sarcopenia were risk factors of umbilical PSH. Also, DR might be a possible risk factor of umbilical PSH occurrence and recurrence. Surgeons should be aware of the presence of DR before the planning of the laparoscopic surgery by diagnostic imaging. If DR is associated with umbilical PSH, we need to consider the correction of both pathologies at the same time.
\end{abstract}

Received September 23, 2019

Revised December 3, 2019

Accepted December 23, 2019

Corresponding author

Ji-Young Sul

Department of Surgery, Chungnam

National University College of

Medicine, 282 Munhwa-ro, Jung-gu,

Daejeon 35015, Korea

Tel: +82-42-280-7175

Fax: +82-42-257-8024

E-mail: jysul@cnu.ac.kr

ORCID:

https://orcid.org/0000-0001-6634-9481

Keywords: Diastasis recti, Port site hernia, Trocar site hernia, Incisional hernia

This is an Open Access article distributed under the terms of the Creative Commons Attribution Non-Commercial License (http:// creativecommons.org/licenses/by-nc/4.0/) which permits unrestricted non-commercial use, distribution, and reproduction in any medium, provided the original work is properly cited.
Copyright (c) 2020 The Journal of Minimally Invasive Surgery. All rights reserved.

\section{INTRODUCTION}

Port site hernia (PSH), also known as port-site incisional hernia (PIH) or trocar site hernia (TSH), is defined as the development of a hernia at the cannular insertion sites, and these terms have been used interchangeably in many articles. The incidence of PSH has varied from $0.65 \sim 6.0 \% .^{1-3}$

Diastasis recti (DR), also known as Diastasis rectus abdominis muscle (DRAM), Rectus diastasis (RD), or divarication of the rectus muscles, is an acquired condition in which the rectus muscles are separated by an abnormal distance along their length, but with no fascial defect. There is no consensus as to the definition of DR, but a separation of $>2 \mathrm{~cm}$ is gener- ally considered to be a rectus diastasis. ${ }^{4}$ The DR studies were mostly done in women, especially in the postpartum period. ${ }^{3}$

Recently, as the population of obesity, the elderly, or the elderly with sarcopenic obesity increases in Korea, the incidence of DR in these population seems to increase as well. Accordingly, its clinical importance should be reappraised because the thinning and stretching of the linea alba is a significant risk factor for the actual development of midline hernias (umbilical, epigastric, trocar, incisional hernia).,

Herein, we describe our experiences with umbilical PSH and concomitant DR in adult patients and would like to raise awareness of the possible role of DR as one of the risk factors of primary or recurrent umbilical PSH. 


\section{MATERIALS AND METHODS}

\section{Diagnostic criteria and classification of DR}

There is no consensus of diagnostic criteria of DR. However, there is a multidisciplinary study about diastasis recti, by anatomo-radiological, and biochemical approach to clarify the normal range of linea alba.

We measured the IRD at the level of the umbilicus according to the Rath classification (Table 1).

\section{Diagnosis and classification of umbilical PSH}

Crist and Gadacz defined port site hernia as the development of a hernia at the canula insertion site. Tonouchi gave a classification system for PSH in 2004, based on the extent of defect and timing of presentation - 3 types 1) early-onset type: dehiscence of the anterior and posterior fascial plane and peritoneum, 2) late-onset type: dehiscence of the anterior and posterior fascial plane. Peritoneum constitutes hernia sac, 3) special type: dehiscence of the whole abdominal wall. The protrusion on intestine and/or omentum. ${ }^{2}$

\section{Data collection}

Between January 2011 and September 2019, the data of 18 patients, who presented with primary or recurrent umbilical PSH after laparoscopic abdominal surgery, was collected and retrospectively analyzed. To demonstrate the association of DR with umbilical PSH, preoperative CT was analyzed and recorded the Inter-recti distance (IRD) for all the patients. Demographic and clinical details were registered (Table 2). Age, gender, BMI, and the presence of sarcopenia were recorded and analyzed. Age and BMI were measured at the time of the first laparoscopic surgery if available. In the evaluation of preexisting co-morbidities, cardiac diseases included the presence of hypertension, valvular heart disease, and coronary artery disease. Pulmonary diseases included COPD or asthma or any

Table 1. The Rath classification based on the level of the attenuation relative to the umbilicus and patient age

\begin{tabular}{|c|c|c|c|}
\hline & \multicolumn{3}{|c|}{ IRD } \\
\hline & Supraumbilicus & Umbilicus & Subumbilicus \\
\hline \multicolumn{4}{|c|}{ Age (years) } \\
\hline$\leq 45$ & $>10 \mathrm{~mm}$ & $>27 \mathrm{~mm}$ & $>9 \mathrm{~mm}$ \\
\hline$>45$ & $>15 \mathrm{~mm}$ & $>27 \mathrm{~mm}$ & $>14 \mathrm{~mm}$ \\
\hline
\end{tabular}

$\mathrm{IRD}=$ inter-recti distance. pulmonary dysfunction that need medical treatment. WHO classification of BMI for Asian was used for classification of obesity. ${ }^{8}$ Sarcopenia criteria based on the Psoas Muscle Index (PMI) at the L3 level by CT scan has been used as a surrogate marker of skeletal muscle mass. Hamaguchi et al. proposed the cutoff values of PMI to define sarcopenia as $6.36 \mathrm{~cm}^{2} / \mathrm{m}^{2}$ for males and $3.92 \mathrm{~cm}^{2} / \mathrm{m}^{2}$ for females in Asian populations. ${ }^{9}$

Table 2. Patient demographics

\begin{tabular}{|c|c|}
\hline & Total $(n=18$ \\
\hline \multicolumn{2}{|l|}{ Age (years) } \\
\hline Mean $\pm S D$ & $64.9 \pm 12.9$ \\
\hline \multicolumn{2}{|l|}{ Gender, N $(\%)$} \\
\hline Male & $8(44.4)$ \\
\hline Female & $10(55.6)$ \\
\hline \multicolumn{2}{|l|}{ BMI $\left(\mathrm{kg} / \mathrm{m}^{2}\right)$} \\
\hline Mean $\pm S D$ & $26.6 \pm 3.1$ \\
\hline Low weight $\mid<18.5)$ & $0(0)$ \\
\hline Normal (18.5 22.9) & $1(5.6)$ \\
\hline Overweighted (23 27.5) & $11(61.1)$ \\
\hline Obese (>27.5) & $6(33.3)$ \\
\hline Sarcopenia (N) & 9 \\
\hline $\mathrm{DR}, \mathrm{N}(\%)$ & $10(55.6)$ \\
\hline \multicolumn{2}{|l|}{ Type of hernia, $N(\%)$} \\
\hline Type 1 & $0(0)$ \\
\hline Type 2 & $18(100)$ \\
\hline Type 3 & $0(0)$ \\
\hline Port extension, $\mathrm{N}(\%)$ & $10 \mid 55.6)$ \\
\hline \multicolumn{2}{|l|}{ Interval ${ }^{*}$ (months) } \\
\hline Mean $\pm S D$ & $41.2 \pm 83.9$ \\
\hline Infection, N $(\%)$ & $2(11.1)$ \\
\hline Referred $^{\dagger}, \mathrm{N}(\%)$ & 6 (33.3) \\
\hline \multicolumn{2}{|l|}{ Co-morbidities, N $|\%|$} \\
\hline Cardiac disease & $9(50)$ \\
\hline Pulmonary disease & $1(5.6)$ \\
\hline $\mathrm{DM}$ & $5(27.8)$ \\
\hline Cancer & $11(61.1)$ \\
\hline
\end{tabular}




\section{Characteristics between the DR and the non-DR group patients}

The patients were divided into the two groups into the DR and the non-DR groups and compared with the medical information (Table 3). The trocar size of the umbilicus was all more than $1.2 \mathrm{~cm}$, so we reviewed the extent of port incision, instead. Also, wound infection, types of operation, and suture materials were recorded and analyzed if they were available. The interval between the first laparoscopic surgery and diagnosis of PSH was calculated as months.

\section{Statistical analysis}

All quantitative results were expressed as mean \pm standard deviation. Mann-Whitney U-test was used for a statistical significance test. A 2-tailed $p>0.05$ was considered statistically significant. All statistical analysis was performed using SPSS software (version 23; SPSS Inc., Chicago, IL).

This study was approved by the Institutional Review Board of Chungnam National University Hospital.

\section{RESULTS}

There were eighteen patients with umbilical PSH during the study period. Among them, six patients were referred from another hospital. In our facility, there were total 10,179 minimally invasive surgeries during the same period of the study, so the incidence of the umbilical PSH was $0.12 \%$.

All our patients were Type 2 late-onset type PSH, which occurred several months or more after the first laparoscopic surgery, and they were presented with periumbilical bulging with no intestinal obstruction.

Ten out of eighteen patients with umbilical PSH had DR

Table 3. Characteristics of the DR and the non DR patient group

\begin{tabular}{|c|c|c|c|c|}
\hline & Total $(n=18)$ & $\mathrm{DR}(\mathrm{n}=10)$ & Non DR $(n=8)$ & $p$ value \\
\hline \multicolumn{5}{|c|}{ Hernia defect size (cm), N (\%) } \\
\hline $0 \sim 1.9$ & & $1(10)$ & $1(12.5)$ & \\
\hline $2 \sim 3.9$ & & $4(40)$ & $5(62.5)$ & \\
\hline $4 \sim 5.9$ & & $3(30)$ & $1(12.5)$ & \\
\hline$\geq 6$ & & $2(20)$ & $1(12.5)$ & \\
\hline Mean $\pm S D$ & $4.1 \pm 1.8$ & $4.5 \pm 1.9$ & $3.6 \pm 1.5$ & 0.263 \\
\hline \multicolumn{5}{|l|}{$\mid R D^{*}(\mathrm{~cm}), \mathrm{N}(\%)$} \\
\hline Mean $\pm S D$ & $3.20 \pm 1.27$ & $3.98 \pm 1.2$ & $2.23 \pm 0.33$ & \\
\hline Port extension, $\mathrm{N}(\%)$ & 10 & $7(70)$ & $3(27.5)$ & 0.829 \\
\hline \multicolumn{5}{|l|}{ Interval (months) } \\
\hline Mean $\pm S D$ & $41.2 \pm 83.9$ & $61.1 \pm 110.2$ & $16.4 \pm 13.7$ & 0.315 \\
\hline \multicolumn{5}{|l|}{ Types of operation, N } \\
\hline Cholecystectomy & 2 & 1 & 1 & \\
\hline Colo-rectal resection & 8 & 7 & 1 & \\
\hline Gastric resection & 2 & 2 & 0 & \\
\hline BSO & 2 & 0 & 2 & \\
\hline Hepatic resection & 1 & 0 & 1 & \\
\hline Nephrectomy & 2 & 2 & 0 & \\
\hline Fundoplication & 1 & 0 & 1 & \\
\hline Wound infection, N (\%) & 2 & $2(20)$ & $0(0)$ & 0.192 \\
\hline Sarcopenia & 9 & $6(60)$ & $3(37.5)$ & \\
\hline Recurred hernia, $\mathrm{N}(\%)$ & 5 & $4(40)$ & $1(12.5)$ & 0.208 \\
\hline
\end{tabular}

$\mathrm{DR}=$ diastasis recti; $\mathrm{SD}=$ standard deviation; IRD = inter-recti distance; $\mathrm{BSO}=$ bilateral salphingo-oophorectomy. ${ }^{*}$ Time between initial laparoscopic surgery to first herniorrhaphy. 
Table 4. Clinical review of recurrent umbilical hernia

\begin{tabular}{|lccccc}
\hline \multicolumn{1}{c}{ Patient } & A & B & C & D & E \\
\hline Age & 65 & 76 & 62 & 38 & 39 \\
Gender & F & M & F & F & F \\
\hline BMI & 30.31 & 22.49 & 24.75 & 26.12 & 29.7 \\
\hline DR & Yes & Yes & Yes & No & Yes \\
\hline IRD (cm) & 6 & 3.4 & 3.9 & - & 5.4 \\
\hline Port extension (cm) & $1.5(4)$ & $1(2)$ & 1 & $1(2)$ & 1 \\
\hline Defect size (cm) & 5.7 & 7.9 & 3.4 & 1.7 & 5.3 \\
\hline Sarcopenia & Yes & Yes & Yes & No & No \\
\hline Wound infection & Yes & No & Yes & No & No \\
\hline Recurrence (times) & 4 & 3 & 2 & 2 & 3 \\
\hline
\end{tabular}

$\mathrm{DR}=$ diastasis recti; $\mathrm{IRD}=$ inter-recti distance.

before the first laparoscopic abdominal surgery.

There was a trend toward higher BMI, the presence of DR, the port extension, and the presence of sarcopenia being associated with the more number of umbilical PSH patients, though this was not seen statistically due to the small number of patient samples.

In the DR group, an abnormally wide IRD was present. Higher incidence of sarcopenia in the DR group (60\% vs. $37.5 \%$ ) implies that the anatomopathological features of sarcopenia can affect DR. It is noteworthy that 4 out of 5 recurrence patients had DR, and more than two recurrences were all associated with DR.

The extension of the port incision may be able to cause more PSH in the DR group than the non-DR group (70\% vs. 27.5\%).

Among five patients who had experienced the recurrence or re-recurrence of umbilical PSH, four patients also had DR concomitantly (Table 4).

Seven patients of the DR group (70\%) and three patients of the non-DR group (37.5\%) were female.

However, the incidence of recurrence (40\%), wound infection $(20 \%)$, and sarcopenia $(60 \%)$ were more common in the DR group, although the small sample sizes make it harder to find statistical significance.

Half of umbilical PSH patients had sarcopenia, with a relatively higher incidence in the DR group (60\% vs. $37.5 \%)$.

\section{DISCUSSION}

There is no consensus as to the definition of DR, but a separation of $>2 \mathrm{~cm}$ is generally considered to be a rectus diastasis. ${ }^{4}$ It is well known that age, body mass index (BMI), multiparity, sarcopenia, and gender difference are typical of the risk factors for DR. ${ }^{10}$ The studies of DR have predominantly been focused on pregnant or multipara females, but recently, the incidence of DR is increasing due to the increase in the population of elderly, the obesity, the sarcopenic obesity.

The incidence of PSH is relatively low, about $0.6 \sim 6 \%$, but it is becoming increasing as a result of the escalating number of laparoscopic abdominal surgery. ${ }^{11}$ Midline umbilical PSH is about three times more common than lateral port site hernia following laparoscopic abdominal surgery. ${ }^{5,6}$

The umbilical port site is located in the midline and mostly large more than $1.2 \mathrm{~cm}$, and also frequently extended to retrieve the surgical specimen. So, the umbilicus may well be the most prevalent site of incisional hernia following laparoscopic surgery.

Various risk factors, such as Large trocar size, mid-line trocars, wound infection, wound extension for organ retrieval, pre-existing umbilical defects, or pre-existing diseases have been implicated in the development of PSH, but there are not many reports about the relationship between the midline or umbilical PSH and DR.

In our study, there was a trend toward higher BMI, the presence of $\mathrm{DR}$, the port extension, and the presence of sarcopenia being associated with the more number of umbilical PSH patients, though this was not seen statistically due to the small number of patient samples (Table 2).

For the comparison, the umbilical PSH patients were divided into the two groups, the DR and the non-DR, according to the presence or absence of DR. In the DR group, an abnormally wide IRD was present. Higher incidence of sarcopenia in the DR group (60\% vs. $37.5 \%)$ implies that the anatomopathological features of sarcopenia can affect DR. It is noteworthy that 4 out of 5 recurrence patients had DR, and more than two recurrences were all associated with DR. The extension of the port incision may be able to cause more PSH in the DR group than the non-DR group (70\% vs. $27.5 \%)$.

Diastasis recti (widened linea alba) itself is not clinically significant, and operative repair is not required if there is no symptoms and signs. However, if the gradual thinning and stretching or widening of the linea alba is combined with obesity, sarcopenia, or muscle weakness, then it causes further protrusion and increases the perimeter and tension of the anterior abdominal wall due to general laxity of the ventral abdominal wall muscles. ${ }^{11}$ Also, if DR is combined with port extension and poor wound closure techniques, it may well cause incisional hernia of midline abdominal wall.

The axial CT scans (the initial \& at the time of diagnosis of umbilical PSH) of the patient who had multiple recurrences were shown in Fig. 1. The patient initially had DR and small umbilical hernia before the initial laparoscopic operation (Fig. 

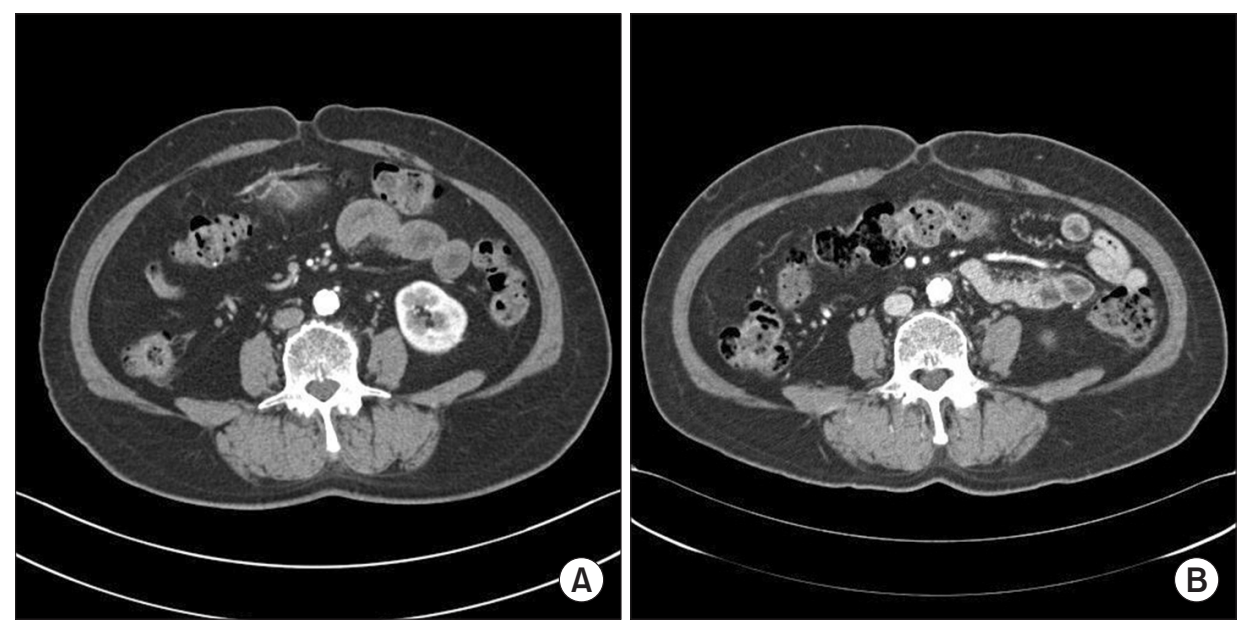

Fig. 1. Preoperative CT scan (A) and aggravated umbilical hernia after primary laparoscopic surgery (B).
1A). The surgeon at the time did not recognize the presence of DR and made a suture repair. As a result, the patient had to have multiple recurrences (three times) of umbilical PSH. Sometimes the surgeon does not recognize the presence of DR before the midline abdominal operation and even after the development of umbilical PSH. ${ }^{3}$ That can be the cause of recurrence and re-recurrence.

So the surgeon should be aware of DR before the planning of the operation, such as where to put the trocars and how to repair port sites.

Though DR-related umbilical PSH is not associated with the patients mortality, it can cause repetitive surgeries, cosmetic problems, and decreased quality of life. Therefore, it may need to investigate all abdominal operation patients with high-risk factors of diastasis recti, such as obesity, large abdomen, elderly, sarcopenia, or the patient with midline incisional hernia regardless of its size, to discover the presence of DR.

When we close the umbilical incision in the patient with DR, it is better to use slow-absorbing absorbable sutures or nonabsorbable sutures. The $5 / 8$ or $1 / 2$ circle needle with polyglycolic acid for closing trocar sites is fast absorbable suture materials, and its absorption rate is more rapid than other slowabsorbing sutures such as polydioxanone, which has more prolonged wound support and guaranteed holding power. ${ }^{11}$ Since DR patient has thinner and easily tearable linea alba, we should use meticulous techniques and slow-absorbable suture materials at least, because fascia or aponeurosis wound healing is slower than any other tissue. Also, with increased protrusion and perimeter of the anterior abdominal wall in the patient with DR, the abdominal wall tension would be increased, so with time, the sutures cannot maintain the holding power of the fascia or linea alba. Therefore, the central concept of incisional hernia prevention of DR patients should include two things; the one is overlap or plication closure of posterior rectus sheaths or midline linea alba with slow-absorbing sutures or non-absorbable sutures and another one is mesh placement to bolster the weak connective tissue. ${ }^{12,13}$ It will reduce the $\mathrm{ab}^{-}$ dominal diameter to lead the decreased outward tensile force on the wound.

In conclusion, all these results may imply that DR may not be the only cause for umbilical PSH, but there might be an association between umbilical PSH and DR. We should consider DR as the significant possible contributing factor of umbilical port site hernia. Although the data didn't reach statistical significance due to the very limited number of patients, more research is needed to confirm and further explore these relationships. In the meantime, as a general surgeon, we should check the status of abdominal wall anatomy preoperatively and should choose the right approach and closure methods among options. If patients have both pathologies, DR and midline hernia, then we should correct both at the same time.

\section{ORCID}

Hyun Jeong Ki, https://orcid.org/0000-0001-6524-8232

Jun Beom Park, https://orcid.org/0000-0002-4804-5254

Ji-Young Sul, https://orcid.org/0000-0001-6634-9481

\section{AUTHORS' CONTRIBUTIONS}

Conceptualization: Ji-Young Sul. Formal analysis: Hyun Jeong Ki. Methodology: Hyun Jeong Ki, Jun Beom Park. Writing-original draft: Hyun Jeong Ki, Jun Beom Park. Writing-review and editing: Ji-Young Sul.

\section{CONFLICT OF INTEREST}

None. 


\section{FUNDING}

None.

\section{ACKNOWLEDGMENTS}

None.

\section{REFERENCES}

1) Rao P, Ghosh K, Sudhan D. Port Site Hernia : A Rare Complication of Laparoscopy. Med J Armed Forces India 2008;64:187-188.

2) Tonouchi H, Ohmori Y, Kobayashi M, Kusunoki M. Trocar site hernia. Arch Surg 2004;139:1248-1256.

3) Mommers EHH, Ponten JEH, Al Omar AK, de Vries Reilingh TS, Bouvy ND, Nienhuijs SW. The general surgeon's perspective of rectus diastasis. A systematic review of treatment options. Surg Endosc 2017;31:4934-4949.

4) Reinpold W, Köckerling F, Bittner R, et al. Classification of Rectus Diastasis-A Proposal by the German Hernia Society (DHG) and the International Endohernia Society (IEHS). Front Surg 2019;6:1.

5) Lambertz A, Stüben BO, Bock B, et al. Port-site incisional herniaA case series of 54 patients. Ann Med Surg (Lond) 2017:14:8-11.
6) Memon MR, Arshad S, Rafiq S, Bozdar AG, Shah SQA. Port-site hernia: A serious complication of laparoscopy. Rawal Med J 2011; 36:14-17.

7) Rath AM, Attali P, Dumas JL, Goldlust D, Zhang J, Chevrel JP. The abdominal linea alba: an anatomo-radiologic and biomechanical study. Surg Radiol Anat 1996;18:281-288.

8) Kim MK, Lee WY, Kang JH, et al. 2014 clinical practice guidelines for overweight and obesity in Korea. Endocrinol Metab (Seoul) 2014;29:405-409.

9) Hamaguchi $Y$, Kaido T, Okumura S, et al. Proposal for new diagnostic criteria for low skeletal muscle mass based on computed tomography imaging in Asian adults. Nutrition 2016;32:1200-1205.

10) Pulle MV, Siddhartha R, Dey A, Mittal T, Malik VK. Port site hernia in laparoscopic surgery: Mechanism, prevention and management. Curr Med Res Pract 2015;5:130-137.

11) Nahas FX, Augusto SM, Ghelfond C. Nylon versus polydioxanone in the correction of rectus diastasis. Plast Reconstr Surg 2001;107: 700-706.

12) Doubkova L, Andel R, Palascakova-Springrova I, Kolar P, Kriz J, Kobesova A. Diastasis of rectus abdominis muscles in low back pain patients. J Back Musculoskelet Rehabil 2018;31:107-112.

13) Nahas FX, Ferreira LM, Mendes JdA. An efficient way to correct recurrent rectus diastasis. Aesthetic Plast Surg 2004;28:189-196. 